\title{
Dark Energy Is a Phenomenal Effect of the Expanding Universe-Possibility for Experimental Verification*
}

\author{
Siva Prasad Kodukula (1) \\ Independent Researcher, Visakhapatnam, India \\ Email: sivkod@gmail.com
}

How to cite this paper: Kodukula, S.P. (2021) Dark Energy Is a Phenomenal Effect of the Expanding Universe-Possibility for Experimental Verification. Journal of High Energy Physics, Gravitation and Cosmology, 7, 1333-1352.

https://doi.org/10.4236/jhepgc.2021.74083

Received: July 27, 2021

Accepted: October 9, 2021

Published: October 12, 2021

Copyright $\odot 2021$ by author(s) and Scientific Research Publishing Inc. This work is licensed under the Creative Commons Attribution-NonCommercial International License (CC BY-NC 4.0).

http://creativecommons.org/licenses/by-nc/4.0/

\begin{abstract}
Vacuum energy density has been defined and mass formation from "space-time" has been viewed from a different perspective. This explanation for vacuum energy is based on "space-time" and conversion of space into time keeping "space-time density" as constant. Equations for "space-time" and mutual conversion of space and time have been derived. As a result, new concept of mass creation has been explained. By postulating that space time density of universe is constant, low and high values of cosmological constant has been shown as the exchange of energy between space, time and matter. The concept has been used to explain dark energy concept of the universe. It concluded a result that velocity of light is changing with the apparent expansion of the universe. The derived equation is possible for experimental verification. Obviously it is a contradiction to Big bang model. So the derived equation with the help of quantum concepts explained the $2.7 \mathrm{~K}$ cosmic micro wave background radiation. Theoretical value of Hubble's constant has been proposed which is useful for calculation of experimental results appropriately.
\end{abstract}

\section{Keywords}

Space-Time, Vacuum Energy, Dark Energy, Velocity of Light, Hubble's Constant, Universe

\section{Introduction}

Fundamentally mass creation has been explained from the classical physics based on the fact that vacuum energy is due to space time dynamics and conversion of space into time keeping space time density constant. A new concept has been in${ }^{\star}$ This publication is a revised/publication version of preprint: https://doi.org/10.21203/rs.3.rs-40479/v2. 
troduced. According to that, space time will be contracted to form a mass. Every mass will have its own space time. Each and every space time will have a quantum particle. Quantum particle of our four dimensional space time is Planck particle. It is explained that space time is not expanding. It has existed with Planck diameter. But due to conversion of space into time, it seems that time is increasing but here space contracting so that space time density is constant. So as per observation it seems that universe is expanding and the energy density when it is at size of plank diameter will remain constant even apparent expansion of the universe reaches maximum velocity as per Hubble's law. Actually the variation in mass energy density has been termed as cosmological constant catastrophe in cosmology and explained in various papers [1]. Few more results have been achieved by using concepts "Siva's gravity equation" and its application to expanding universe which was elaborated in the paper [2] [3]. Of course the main difference is that space expands with time where as this paper emphasizes that space time is a continuum and $3 \mathrm{D}$ space and $4 \mathrm{D}$ space are same. Space time is a combination of space and time. Time decreases with the expansion of space. As a consequence the mass energy will be increased. The variation in mass energy density of vacuum catastrophe in cosmology is a consequence of this phenomenon.

\section{Concept and Calculation}

Let us start with classical explanations for space, time, space time, mass and their relation. This will be initiated from some postulates. Concept of black holes have been explained with the use of Siva's constant " $K$ " and related gravity equation [3]. How a black hole singularity will emerge as a particle has been explained by an equation relating singularity, mass of black hole and Siva's constant " $K$ ". Space time conversion and Siva's classical equations have been used.

\section{Postulates}

1) Surface area of Space time in spherical shape is volume of its space and radius is its time.

2) Space and time are interchangeable in an isolated system of space time in such a way that reduction in time will compensate in increase of its surface area so that the volume of space will increase if time decreases in space time. Thus the total space time diameter will remain constant.

3) It is based on the assumption that space time is like a fluid and its contraction towards its centre point creates the mass. It is spherical in shape since space time will be curved around a mass. It is an isolated system so totally it will be in spherical shape.

Mathematically,

$$
\begin{aligned}
\frac{4}{3} \pi t^{3} & =4 \pi d^{2} \\
t^{3} & =3 d^{2}
\end{aligned}
$$

$$
\therefore t=1.44224957 \times d^{2 / 3} \text { and }
$$




$$
d=\sqrt{\left(\frac{t^{3}}{3}\right)}
$$

We have Siva's gravity equation [2]

$$
K=G t_{p}\left(\frac{M}{d}\right)
$$

" $t_{p}$ " is plank time so it can be converted in to a space of with a radius " $d$ ".

$$
K=G \times 1.44224957 \times d^{2 / 3}\left(\frac{M}{d}\right)
$$

" $d$ " in bracket is diameter " $d$ " outside bracket is radius.

So convert the outside " $d$ " in to diameter and substitute $d / 2$ in place of " $d$ ". The equation can be rewrite as

$$
K=G \times 1.44224957 \times\left(\frac{d}{2}\right)^{2 / 3}\left(\frac{M}{d}\right)
$$

This is a generalized equation for all space times with different values of " $K$ ". For our visible universe, $K=4.84533 \times 10^{-27} \mathrm{~m}^{2} \cdot \mathrm{sec}^{-1}$ [3].

So for this universe or for gravity space time, Equation (6) can be written as

$$
\begin{gathered}
4.84533 \times 10^{-27}=6.67408 \times 10^{-11} \times 1.44224957 \times\left(\frac{d}{2}\right)^{2 / 3}\left(\frac{M}{d}\right) \\
4.84533 \times 10^{-27}=\frac{6.67408 \times 10^{-11} \times 1.44224957}{\left(2^{2}\right)^{\frac{1}{3}} \times d^{\frac{1}{3}}} \times M \\
d^{1 / 3}=\frac{6.67408 \times 10^{-11} \times 1.44224957}{\left(2^{2}\right)^{\frac{1}{3}} \times 4.84533 \times 10^{-27}} \times M \\
d^{1 / 3}=\frac{6.67408 \times 10^{-11} \times 1.44224957}{1.587401 \times 4.84533 \times 10^{-27}} \times M \\
d^{1 / 3} \times \frac{1.587401 \times 4.84533 \times 10^{-27}}{6.67408 \times 10^{-11} \times 1.44224957}=M \\
M= \\
=\frac{1.587401 \times 4.84533 \times 10^{-27}}{6.67408 \times 10^{-11} \times 1.44224957} \times d^{1 / 3} \\
M=7.9905778 \times 10^{-17} \times d^{1 / 3}
\end{gathered}
$$

We know the density $\gamma=\frac{m}{v}$

$$
\begin{gathered}
\gamma=\frac{M}{\frac{4}{3} \pi r^{3}} \\
\gamma=\frac{7.9905778 \times 10^{-17} \times d^{1 / 3}}{\frac{4}{3} \pi\left(\frac{d}{2}\right)^{3}}
\end{gathered}
$$




$$
\begin{gathered}
\gamma=\frac{3 \times 8 \times 7.9905778 \times 10^{-17} \times d^{1 / 3}}{4 \pi d^{3}} \\
\gamma=\frac{3 \times 8 \times 7.9905778 \times 10^{-17} \times d^{1 / 3}}{4 \pi d^{3}} \\
\gamma d^{8 / 3}=\frac{3 \times 8 \times 7.9905778 \times 10^{-17}}{4 \pi} \\
\therefore \gamma d^{8 / 3}=1.526087946 \times 10^{-16}
\end{gathered}
$$

\section{Quantum Vacuum \& Cosmological Catastrophe}

As per the predictions of quantum mechanics, the vacuum energy is $10^{123}$ orders of the mass energy density of the universe. This huge discrepancy between quantum mechanics and mass energy density calculated due to expansion of the universe is called as cosmological catastrophe described in paper [1].

Let us calculate the same by classical approach by using new perspective of expanding universe [3].

1) According to that, at a point of time the diameter of the universe is equal to diameter of Planck length $1.616229 \times 10^{-35} \mathrm{~m}$ [4]. So its volume is

$$
\frac{4}{3} \pi r^{3}=\frac{4 \times \pi\left(1.616229 \times 10^{-35}\right)^{3}}{3 \times 2^{3}}=2.2105854514 \times 10^{-105} \mathrm{~m}^{3}
$$

Its mass is Planck mass [4] i.e. $2.176470 \times 10^{-8} \mathrm{~kg}$

$$
\begin{aligned}
& \begin{aligned}
& \therefore \text { Energy }=m c^{2}= 2.176470 \times 10^{-8} \times\left(2.99792458 \times 10^{8}\right)^{2} \mathrm{~J} \\
&=1.9561136839 \times 10^{9} \mathrm{~J}
\end{aligned} \\
& \begin{aligned}
\therefore \text { Energy density } & =\frac{\text { energy }}{\text { volume }}=\frac{1.9561136839 \times 10^{9}}{2.2105854514 \times 10^{-105}} \\
& =8.84884899 \times 10^{113} \mathrm{~J} \cdot \mathrm{m}^{-3}
\end{aligned}
\end{aligned}
$$

This vacuum Energy density is higher value and is based on Planck scale defined by quantum mechanics.

2) For vacuum, the space time conversion will affect its mass also. Mass of the universe [3] has been calculated as $1.7898298 \times 10^{53} \mathrm{~kg}$. Now the vaccum energy density can be calculated as follows:

By Hubble's equation maximum diameter of the Universe $d=\frac{H}{C}$.

Where " $H$ " is Hubble's constant [5] [6] and " $c$ " is velocity of light.

$$
\begin{gathered}
d=\frac{c}{H}=\frac{2.99792458 \times 10^{8}}{2.255582386 \times 10^{-18}}=1.3291133140 \times 10^{26} \mathrm{~m} \\
\frac{4}{3} \pi\left(\frac{d}{2}\right)^{3}=\frac{4 \times \pi\left(1.3291133140 \times 10^{26}\right)^{3}}{3 \times 2^{3}}=1.2293757654 \times 10^{78} \mathrm{~m}^{3}
\end{gathered}
$$

At this stage mass of the Universe [3] is $1.7898298 \times 10^{53} \mathrm{~kg}$ 


$$
\begin{gathered}
\text { Energy }=m c^{2}=1.7898298 \times 10^{53} \times\left(2.99792458 \times 10^{8}\right)^{2} \mathrm{~J} \\
=1.6086188018 \times 10^{70} \mathrm{~J} \\
\text { Energy density }=\frac{\text { energy }}{\text { volume }}=\frac{1.6086188018 \times 10^{70}}{1.2293757654 \times 10^{78}}=1.30848423 \times 10^{-8} \mathrm{~J} \cdot \mathrm{m}^{-3}
\end{gathered}
$$

This vacuum Energy density is very low value and is based on the volumetric expansion of the space. Mass is "mass of the universe" at its maximum volume.

Above two cases explains upper and lower energy density $8.884884899 \times 10^{113}$ $\mathrm{J} \cdot \mathrm{m}^{-3}$ and $1.30848423 \times 10^{-8} \mathrm{~J} \cdot \mathrm{m}^{-3}$.

It is similar to the calculation of quantum catastrophe by others [1].

Here the velocity of light " $c$ " is $2.99792458 \times 10^{8} \mathrm{~m}[4]$ and "Hubble's constant" " $H$ " is $2.255582386 \times 10^{-18} \mathrm{sec}^{-1}$ [5] [6]. It seems that these values are not correct values to propose a perfect model. Further sections will elaborate that it is necessary to correct the values of " $c$ " and " $H$ ". These exact values must be used in mass energy density calculation and the above calculations will be revised further for a perfect solution.

\section{Analysis on This Cosmological Catastrophe}

If we apply time conversion in to space and space time conversion in to mass, the calculation for above two cases i.e., lower and upper values of energy density can be viewed as a two different out comes of a single phenomenon. In quantum level it can be explained as follows:

- All the fundamental forces are basically made up of quantum particles [7] quantum of electromagnetic force is photon and for gravitational force is graviton.

- All these quantum particles are made up of space time and contains space time diameter depending upon their coupling constant [7].

- The quantities like mass, space time diameter associated to a quantum particle are invariant. They will remain constant. But the complete universe is filled with these quantum particles. If the universe (at the beginning) is with the diameter of space time of the quantum particle, there exist only one particle and as the space increases, the number of quantum particles will increase and the space time density will be maintained as constant.

- As the number of particles increases, total mass will be increased. As per energy equivalent principle, energy neither can be increased nor destroyed. Implies mass will be increased if the term ' $d$ ' in Equation (4) decreases. ' $d$ ' decreases means space decreases. But each and every such field will have a Siva's constant ' $K$. For our four dimensional space time ' $K$ ' value [3] is $4.84533 \times 10^{-27} \mathrm{~m}^{2} \cdot \mathrm{sec}^{-1}$. Thus some portion of space time will be converted in to energy or mass and space will be decreased. The other portion of space will be converted in to time and the space time density will be maintained constant by converting in to one another and keeping Sivas constant ' $K$ ' and mass energy density as constants. 
- Thus for both the above cases, the mass energy density is constant. Only observation of time and space seems to be changing. Thus cosmological constant catastrophe can be explained.

- In the same way as explained above, graviton is a fundamental particle with space time dia.1.5466374059 $\times 10^{-47}$. mt. and $K=4.636702 \times 10^{-39} \mathrm{~m}^{2} \cdot \mathrm{sec}^{-1}$. Photon is a fundamental force particle with space time diameter $6.783124 \times$ $10^{-20} \mathrm{mt}$ and $K=2.033529 \times 10^{-11} \mathrm{~m}^{2} \cdot \mathrm{sec}^{-1}$ [7].

If we calculate the two observations as originated by single phenomenon explained by space time energy conversions from one to another by using Equations (2) \& (4), we can calculate the energy density as follows:

$$
\text { Volume }=\frac{4}{3} \pi\left(\frac{d}{2}\right)^{3}=\frac{\pi}{6} d^{3}
$$

We have Equation (4)

$$
\begin{aligned}
& K=G t_{p}\left(\frac{M}{d}\right) \\
& \therefore d=\frac{G t_{p} m}{K}
\end{aligned}
$$

We have Equation (2) for conversion of space and time

$$
\begin{gathered}
t^{3}=3 d^{2} \\
t^{2} t=3 d^{2} \\
t=3\left(\frac{d}{t}\right)^{2} \\
c=\frac{d}{t} \Rightarrow t=3 c^{2} \\
d^{2}=\frac{t^{3}}{3} \\
d^{2}=\frac{\left(3 c^{2}\right)^{3}}{3} \\
\Rightarrow d^{2}=9 c^{6}
\end{gathered}
$$

Volume of space time as per Equation (11) $=\frac{\pi}{6} d^{3}$

$$
\text { volume }=\frac{\pi}{6} d^{3}=\frac{\pi}{6} d^{2} \times d
$$

Substitute Equation (12) and Equation (14) in Equation (24) to find its volume. We have Equation (15),

$$
\begin{gathered}
\text { volume }=\frac{\pi}{6} d^{2} \times d \\
\therefore \text { volume }=\frac{\pi}{6} \times 9 c^{6} \times \frac{G t_{p} m}{K}
\end{gathered}
$$

We know energy $E=m c^{2}$ 
Mass energy density

$$
\begin{gathered}
E_{D}=\frac{m c^{2}}{\frac{\pi}{6} \times 9 c^{6} \times \frac{G t_{p} m}{K}}=\frac{6 m c^{2} K}{\pi G t_{p} m 9 c^{6}} \\
E_{D}=\frac{6 K}{\pi G t_{p} 9 c^{4}} \\
=\frac{6 \times 4.84533 \times 10^{-27}}{\pi \times 6.67408 \times 10^{-11} \times 5.39116 \times 10^{-44} \times 9 \times\left(2.99792458 \times 10^{8}\right)^{4}} \\
=\frac{29.07198 \times 10^{-27}}{8.2176723821 \times 10^{-19}} \\
=3.5377389908 \times 10^{-8} \mathrm{~J} \cdot \mathrm{m}^{-3} \\
\therefore E_{D}=3.5377389908 \times 10^{-8} \mathrm{~J} \cdot \mathrm{m}^{-3}
\end{gathered}
$$

In this we have used $c=2.99792458 \times 10^{8} \mathrm{~m} \cdot \mathrm{sec}^{-1}$ [4]. This value of " $c$ " must be changed for a perfect model of the space time. The above calculation for mass density must be revised according to the changed value of " $c$ ".

\subsection{Is Velocity of Light Changing? What is Its Maximum Velocity?}

In the above calculations we have used that the velocity of light $c=2.99792458 \times 10^{8} \mathrm{~m} \cdot \mathrm{sec}^{-1}$. But light velocity is a significant velocity of theory of relativity which must be constant and maximum velocity used in transformations. But here it is obvious that signal velocity depends on space and time. Let us see how it will be changed with space and time conversions of the universe.

Figure 1 shows the space time expansion of the universe. It is like a sphere with centre $\mathrm{O}$. The radius of the sphere is " $t$ ". " $\mathrm{A}$ " and " $\mathrm{B}$ " are galaxies over its surface. Linear distance between these two galaxies is " $d$ ". We are in the galaxy " $\mathrm{B}$ " and observing the velocity of " $\mathrm{A}$ " receding from galaxy " $\mathrm{B}$ " as explained by expanding universe and follow the equation $V=H d$. As per expanding Universe theory, velocity of the receding galaxy is proportional to distance from the observer and proportionality of the constant is " $H$ ".

$$
\begin{aligned}
& V=H d \\
& \Rightarrow d=\frac{V}{H}
\end{aligned}
$$

As per space time equivalence principle time and distance expressed by Equation (3) i.e.

$$
t^{3}=3 d^{2}
$$

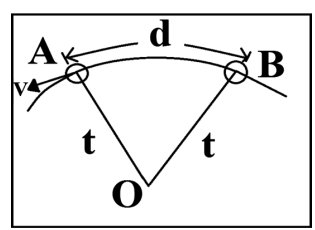

Figure 1. 3D view of 4D space-time Conversion of time for expansion of space (universe). 
Multiply both sides with " $d$ "

$$
\begin{aligned}
& \therefore d t^{3}=3 d^{3} \\
& \therefore d=3\left(\frac{d}{t}\right)^{3}
\end{aligned}
$$

It takes " $t$ " seconds to receive light like signal from A to B, Let us suppose that signal velocity is $c_{0}$. Substitute $c_{0}$ instead of $\frac{d}{t}$ we can rewrite the Equation (19) as

$$
d=3 c_{o}^{3}
$$

We have Equation(18)

$$
\begin{gathered}
d=\frac{V}{H} \\
\therefore V=3 H c_{o}^{3} \\
\therefore c_{o}=\sqrt[3]{\frac{V}{3 H}}
\end{gathered}
$$

So it shows a relation between velocity of light observed from a receding galaxy with velocity " $V$ ". It can be observed experimentally by any astronomical observatory. We can observe the velocity of receding galaxy is $V$ by red shift and Doppler Effect. Thus we can find the velocity of light generated from that receding galaxy. This is against to the present understanding of physics which says that light velocity is constant and is equal to $2.99792458 \times 10^{8} \mathrm{~m} \cdot \mathrm{sec}^{-1}$

\section{Calculation for Maximum Velocity of Light}

We have Equation (21)

$$
V=3 H c_{o}^{3}
$$

Here " $V$ " is maximum velocity and the signal is also maximum velocity " $V$ " only.

So equation can be written as

$$
\begin{gathered}
c_{o}=3 H c_{o}^{3} \\
c_{o}^{2}=\frac{1}{3 H} \\
c_{o}=\sqrt{\frac{1}{3 H}}=\sqrt{\frac{1}{3 \times 2.255582386 \times 10^{-18}}}=3.8442358581 \times 10^{8} \mathrm{~m} \cdot \mathrm{sec}^{-1}
\end{gathered}
$$

In the above calculations, the value of Hubble's constant [5] [6]

$H=2.255582386 \times 10^{-18} \mathrm{sec}^{-1}$. Any change in value of this constant will change the above result.

Most of the astrophysical calculations are based on Hubble's constant. But the Hubble's constant cannot be an exact value since it depends on observational values of receding galaxies. The results are still ambiguous. Are there any exact theoretical values which satisfy this quantum universe? Especially to explain ac- 
curate anticipation of temperature of first photon which was observed as $2.7 \mathrm{~K}$ cosmic microwave back ground radiation. Thus it is a mandatory requirement to find out the significance of theoretical value of Hubble's constant by postulating a relation with diameter of hydrogen atom.

\subsection{Exact Theoretical Value of "Hubble's Constant"}

As per film theory of the universe [8], a film is a graph plotted for the absolute velocity $\frac{\mathrm{V}}{\gamma}$ and the distance " $d$ " from the observer. Further to that concept, a single film of the universe can be interpreted as two forces as shown in Figure 2.

In this 1 denotes expansion and contraction (gravity force) both at the same time represented as $\mathrm{OA}$ and $\mathrm{AC}$ portions of the graph respectively. OA portion is associated to $V=H d$ equation and $\mathrm{AC}$ is associated to $V d=K$ equation. There exist two velocities in opposite directions for a " $d$ " value provided " $H$ " and " $K$ " are constants. Velocity described by distance and time. Here distance is invariant so we can say " $d$ " will have two types of "time" moving in opposite direction. " $d$ " denotes space and will be associated with two directions of time. Thus reality defined as observation by conscious observer is combination of these two. Mathematically we can say it as super position of these two. In quantum mechanics these two directions of time represent two different quantum states describing time. Thus observation of space time defined by physics is a superposition of two quantum states of space times tends to separate in opposite direction. One denotes future and another denotes past and the present is super position of these two quantum states of universal films.

Always, the distance " $d$ " observed by conscious observer is superposition of two quantum states of space time. $V d=K$ represents gravity field. So, the " $d$ " is the minimum diameter of gravity space time i.e. quantum of gravity field.

As explained in section-4, graviton is a fundamental particle with space time diameter [7]. $1.5466374059 \times 10^{-47} \cdot \mathrm{mt}$. and $K=4.636702 \times 10^{-39} \mathrm{~m}^{2} \cdot \mathrm{sec}^{-1}$.

Photon is a fundamental force particle with space time diameter [7] 6.783124 $\times 10^{-20} \mathrm{mt}$ and $K=2.033529 \times 10^{-11} \mathrm{~m}^{2} \cdot \mathrm{sec}^{-1}$.

Plank mass is also a fundamental force particle with " $K$ " value $4.84533 \times 10^{-27}$ $\mathrm{m}^{2} \cdot \sec ^{-1}$ which is our conventional four-dimensional space time.

Here space time diameter of photon is more than planck diameter and the first photon can be calculated based on this to find out the $2.7 \mathrm{~K}$. Space time

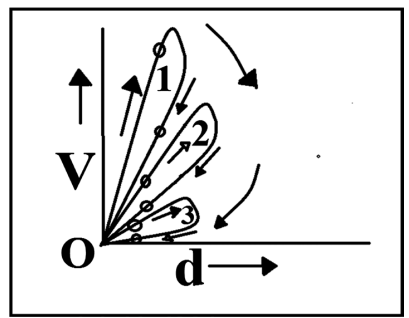

Figure 2. Quantum superposition in films of Universe. 1, 2 \& 3 are films of the Universe Circles denoted on films are quantum states representing the arrow of time. 
diameter of graviton is less than the planck mass. So below the space time diameter of first graviton there will not be gravity. As the space time diameter increases, number of gravitons will increase and gravity will be increased but it can not have the capacity to form neutral matter in this four dimensional universe. The relation between " $K$ " and " $H$ " for gravity space time shows a relation with diameter equal to $1.0583544213 \times 10^{-10} \mathrm{~m}$. This is equal to diameter of hydrogen atom which is neutral atom of first element. The visible matter of the universe is formed by elements. Thus diameter of atom with one single electron is significant in finding the relation between " $K$ " and " $H$ ".

For quantum of gravity field the space time diameter [7] $K=4.636702 \times 10^{-39} \mathrm{~m}^{2} \cdot \mathrm{sec}^{-1}$ and $H=2.255582386 \times 10^{-18} \mathrm{sec}^{-1}$, the " $d$ " is a super position of two space time states.

Let us see the relation between these two equations.

Hubble's equation $V=H d$.

And Siva's equation [2] [8] $V d=K$.

By solving these two equations,

$$
d^{2}=\frac{K}{H}
$$

After substituting " $K$ " and " $H$ " values, we can find a value of " $d$ " nearly equal to hydrogen atom.

Here simply, if we postulate it as equal to diameter of neutral hydrogen atom, we can find the value of " $H$ ". This will be the exact theoretical value of Hubble's constant. Calculation of temperature of first photon which was observed as $2.7 \mathrm{~K}$ cosmic microwave back ground radiation cannot be achieved by light velocity and Hubble's value. $H=2.255582386 \times 10^{-18} \sec ^{-1}$. Already we have calculated the change in light velocity. Still a small change in " $H$ " is also required to achieve this observed result of CMBR. Logical relation between " $K$ " and " $H$ " shows the minimum diameter of space time that keeps super position of two space time states. Below that diameter this superposition will not be maintained and will be divided in to two separate space times in opposite direction. This is the critical limit that denotes the property of matter just before its division. This is nothing but division in to positive and native parts of that matter'. One more reason is 'electrons plays important role in making atom and matter'. Hydrogen is the element that is the first one in that order. And it is neutral and free. Next one is helium with two electrons but its atomic diameter is less than hydrogen atom. It is forcefully reduced its diameter and under pressure. It cannot be said as free. Neutral matter plays important role in universe. So the term neutral is very significant in calculating the minimum diameter of this space time to keep neutral matter.

Any how this assumption gives us $\mathrm{n}$ accurate value of CMBR. So this has become mandatory to postulate that there is a relation between Values of " $K$ ", " $H$ " and diameter of neutral hydrogen atom.

(Space time splits below this diameter. This is because of direction of time. 
Direction of time denotes the charge as positive or negative. So below this diameter positive or negative charges will exist. So this diameter only should be taken. Helium cannot be taken because, if less diameter exist with more electron what is the necessity of existence of element with one electron in this nature?)

We have diameter of Hydrogen atom [4] $d=1.0583544213 \times 10^{-10} \mathrm{~m}$.

Siva's Constant (for gravity) [7] $K=4.636702 \times 10^{-39} \mathrm{~m}^{2} \cdot \mathrm{sec}^{-1}$.

As per Equation (33),

$$
H_{\text {th }}=\frac{K}{d^{2}}=\frac{4.636702 \times 10^{-39}}{\left(1.0583544213 \times 10^{-10}\right)^{2}}=4.1394908592 \times 10^{-19} \mathrm{sec}^{-1}
$$

Hubble's constant $H=2.255582386 \times 10^{-18} \sec ^{-1}$.

Exact value or Theoretical value of Hubble's constant

$$
H_{\text {th }}=4.1394908592 \times 10^{-19} \sec ^{-1}
$$

\subsection{Velocity of Light at Maximum Diameter of the Universe}

Since the Hubble's constant has been revised, the Equation (32) showing the velocity of light at maximum diameter of the universe. In other words, it is the expected maximum velocity of light if we consider the complete universe.

We have Equation (23),

$$
C_{O}=\sqrt{\frac{1}{3 H}}
$$

The equation can be written with Exact value of Hubble's constant

$$
C_{\max }=\sqrt{\frac{1}{3 H_{t h}}}
$$

Substitute Equations (25) in (26),

$$
\begin{aligned}
C_{\text {max }} & =\sqrt{\frac{1}{3 \times 4.1394908592 \times 10^{-19}}} \\
C_{\text {max }} & =8.9735835075 \times 10^{8} \mathrm{~m} \cdot \mathrm{sec}^{-1}
\end{aligned}
$$

where $C_{\max }$ is maximum velocity of light.

\section{A New Model of "Origin of Universe”}

As per the paper "new perspective of expanding universe" [3], the universe is not expanding. It is existed with Planck diameter and apparently seems to expand to Hubble's diameter. Space time diameter is constant. It will not change. Only ratio of the space and time will be changed due to their conversion from one another. Mass also will be increased due to increment in space and keeps the mass energy density of the universe as constant. In this process, "Hubble's constant" and "Siva's constant" will remain constants and both gravity and expansion will exist and follows $V d=K$ and $V=H d$ equations respectively.

As per the quantum mechanics, all the natural forces are made up of quantum particles with specific space time diameters [7]. As explained above these quan- 
tum particles will increase if the volume of space increases.

With the above reasons we can conclude that at time $t=0$, only one quantum particle whose space time diameter is less will exist. Later its number will increase and mass will increase as its time converts in to space. Other forces also will be developed and their interaction creates several elementary particles and the time converts in to space time and then to mass. It exists as it is and its energy density remains constant. Only conversion from one to another will happen. It is against to the "big bang model".

But 2.7 K Cosmic Microwave Background Radiation (CMBR) is the solid experimental proof for Big bang model. Now this CMBR can also be explained by the model which denies the Big bang

\section{Interpretation of Cosmic Microwave Background Radiation (CMBR)}

It is nothing but the energy of single photon.

We have space time diameter for a photon [7] $d=6.783124 \times 10^{-20} \mathrm{~m}$.

This is diameter of the Universe and as per Equation (20)

$$
\begin{gathered}
d=3 c_{o}^{3} \\
\therefore c_{o}^{3}=\frac{d}{3} \\
\therefore c_{o}=\left(\frac{6.783124 \times 10^{-20}}{3}\right)^{\frac{1}{3}} \\
=(22.6104133333)^{1 / 3} \times 10^{-7} \\
=2.8277184609 \times 10^{-7} \mathrm{~m} \cdot \mathrm{sec}^{-1} \\
\therefore c_{o}=2.8277184609 \times 10^{-7} \mathrm{~m} \cdot \mathrm{sec}^{-1}
\end{gathered}
$$

Light velocity is very slow comparable to present measurement.

If we apply wave equation for this velocity with a wavelength equal to this space time diameter, we can find its frequency.

Here light velocity $c_{o}=2.8277184609 \times 10^{-7} \mathrm{~m} \cdot \mathrm{sec}^{-1}$.

Wave length $\lambda=d=6.783124 \times 10^{-20} \mathrm{~m}$.

As per wave equation, Frequency of that wave

$v=\frac{c_{o}}{\lambda}=\frac{2.8277184609 \times 10^{-7}}{6.783124 \times 10^{-20}}=4.1687553714 \times 10^{12} \mathrm{~Hz}$

Energy associated to this single photon $E=m c^{2}$.

Where $m$ is mass of that particle and $c$ is velocity of light i.e. $2.99792458 \times 10^{8}$ $\mathrm{m} \cdot \mathrm{sec}^{-1}$.

We have space time diameter for a photon [7] $d=6.783124 \times 10^{-20} \mathrm{~m}$.

As per siva's classical equation for space time (7) mass

$$
\begin{gathered}
M=7.9905778 \times 10^{-17} \times d^{1 / 3} \\
\therefore m=7.9905778 \times 10^{-17} \times\left(6.783124 \times 10^{-20}\right)^{3} \mathrm{~kg}=3.2587779555 \times 10^{-23} \mathrm{~kg}
\end{gathered}
$$




$$
\begin{gathered}
\therefore E=3.2587779555 \times 10^{-23} \times\left(2.99792458 \times 10^{8}\right)^{2} \mathrm{~J} \\
=2.9288435639 \times 10^{-6} \mathrm{~J} \\
E=2.9288435639 \times 10^{-6} \mathrm{~J}
\end{gathered}
$$

This can be converted to temperature by Stephen-Boltzmann equation

$$
E=\sigma T^{4}
$$

We have Equation (29) for energy

$$
E=2.9288435639 \times 10^{-6} \mathrm{~J}
$$

And [4] $\sigma=5.67036713 \times 10^{-8} \mathrm{~W} \cdot \mathrm{m}^{-2} \cdot \mathrm{K}^{-4}$.

We have equation (30) $E=\sigma T^{4}$

$$
\begin{gathered}
\therefore T=\left(\frac{E}{\sigma}\right)^{0.25}=\left(\frac{2.928843563859 \times 10^{-6}}{5.67036713 \times 10^{-8}}\right)^{0.25}=2.6808421881 \mathrm{~K} \\
T=2.6808421881 \mathrm{~K}
\end{gathered}
$$

Therefore Cosmic Background Radiation (CMBR) temperature is $2.68 \mathrm{~K}$.

\section{Dark Energy is Consequence of a Phenomenon}

Mass energy density calculation used in section-4 as per Equation (18) can be recalculated with correct values of " $c$ " and " $H$ " derived above.

As per Hubble's law, maximum diameter of universe by substituting Equations (25) \& (27),

$$
d=\frac{C_{\max }}{H_{t h}}=\frac{8.9735835075 \times 10^{8}}{4.1394908592 \times 10^{-19}}=2.1677988460 \times 10^{27} \mathrm{~m}
$$

Volume is

$$
\frac{4}{3} \pi\left(\frac{d}{2}\right)^{3}=\frac{4 \times \pi\left(2.1677988460 \times 10^{27}\right)^{3}}{3 \times 2^{3}}=5.3340313589 \times 10^{81}
$$

At this stage mass of the Universe [3] is $1.7898298 \times 10^{53} \mathrm{~kg}$.

" $d$ " changes with " $H$ " so "mass of the universe [3]" must change with the same factor to keep " $K$ " constant in the Equation (4).

$$
\begin{gathered}
\therefore m=1.7898298 \times 10^{53} \times \frac{2.255582386 \times 10^{-18}}{4.1394908592 \times 10^{-19}} \\
\therefore m=9.7526693696 \times 10^{53} \mathrm{~kg} \\
\text { Energy }=m c^{2}=9.7526693696 \times 10^{53} \times\left(8.9735835075 \times 10^{8}\right)^{2} \mathrm{~J} \\
\therefore \text { Energy }= \\
\text { Energy density } E_{D C}=\frac{\text { energy }}{\text { volume }}=\frac{7.85333566094 \times 10^{71}}{5.3340313589 \times 10^{81}} \\
=1.4723116684 \times 10^{-10} \mathrm{~J} \cdot \mathrm{m}^{-3}
\end{gathered}
$$


Mass energy density as per Equation (16),

$$
\begin{aligned}
& =\frac{6 K}{\pi G t_{p} 9 c^{4}} \\
& =\frac{6 \times 4.84533 \times 10^{-27}}{\pi \times 6.67408 \times 10^{-11} \times 5.39116 \times 10^{-44} \times 9 \times\left(8.9735835075 \times 10^{8}\right)^{4}} \\
& =\frac{29.07198 \times 10^{-27}}{6.5967442327 \times 10^{-17}} \\
& =4.4070194287 \times 10^{-10} \mathrm{~J} \cdot \mathrm{m}^{-3}
\end{aligned}
$$

Total mass energy density

$$
E_{D T}=4.4070194287 \times 10^{-10} \mathrm{~J} \cdot \mathrm{m}^{-3}
$$

Energy density calculated

$$
E_{D C}=1.4723116684 \times 10^{-10} \mathrm{~J} \cdot \mathrm{m}^{-3}
$$

Percentage in total mass energy density

$$
\begin{aligned}
& \frac{1.4723116684 \times 10^{-10}}{4.4070194287 \times 10^{-10}}=0.33408331690=33.41 \% \\
& \text { Remaining energy density percentage }=100 \%-33.41 \%=66.59 \%
\end{aligned}
$$

The above calculation concludes that mass energy density is $33.41 \%$ of the total mass energy density including hidden mass energy. The remaining $66.59 \%$ is in the form of space time that which is necessary to produce this mass energy. Expansion is a phenomenon. Evolution of mass is a consequence of this phenomenon. Equation (34) shows the actual total mass energy density of the universe and Equation (35) is the mass energy density of the universe by considering density based on expanding space alone. Thus dark energy is a consequence of missing physics in expanding universe theories. This can be observed experimentally as an effect that changes light velocity with expansion of the universe. This effect can be observed as interpreted by Equation (30). It shows that velocity of light is proportional to the velocity of the receding galaxy calculated by red shift observation.

Finally, in this paper, it is to be noted that concept of four dimensional space time has been taken from General theory of relativity. Curvature of space time in presence of mass also follows general theory of relativity and its equations. This can be viewed as space time continuum as explained in General relativity. This space time continuum explained in General relativity interprets that space and time are separate entities and they coupled in such a way if space increases, time also increase. At this basic level it is contradicted that general relativity is not having any supporting argument that space increases with time. It is assumed based on special relativity concepts. At this basic level It is postulated that space time is a four dimensional material which contains space and time as explained in section-4, mass creation is a consequence of conversion of space and time in to one another. Equation (39) is an experimental verification for this argument which emphasizes concept of "vacuum energy", "dark energy" and resolves "cos- 
mological constant catastrophe".

Physical meaning of space, time and space time continuum are more important than mathematical interpretation of it. Anyhow conclusions leading to the experimental verification are necessary for further advancement of science.

\section{Dark Matter}

We have already calculated the space time diameters for photon and graviton [7]. As per dark energy phenomenology, energy densities calculated for space time diameters of "photon" and "graviton". The relative energy densities have been calculated with reference to space time diameter equivalent to "Planck length". The calculations showed that the energy density of the photon is $6.2 \%$ and invisible energy is remaining $27.21 \%$ (total energy for observation after application of dark energy phenomena is $33.41 \%$ ). Thus the dark matter is $27.21 \%$. Finally we can say dark energy is $66.59 \%$, dark matter is $27.21 \%$ and the visible observable energy is $6.2 \%$.

\section{Review on Few Fruitful Approaches by Others on Dark Energy for a Perfect Probe}

\subsection{Ricci Curvature \& Dark Energy}

Two approaches have been suggested in the paper [9] for dark energy and dark matter approaches. If we look at the dark energy and dark matter problems from General relativities point of view, the right side of equation denotes the dark energy is a new ingredient which caused by large negative pressure which termed as un-clustered non zero vacuum energy and clustered dark matter and it is the standard picture of space time dynamics. The left side of the equation denotes that Dark energy and dark matter are depends on observational aspects involving $f(R)$ theories of gravity, where $R$ is the Ricci curvature scalar, and/or Scalar-Tensor Gravity. On the other hand author suggested that one can define Extended Theories of Gravity those semi classical theories where the Lagrangian is modified, in respect to the standard Einstein-Hilbert gravitational Lagrangian, adding high-order terms in the curvature invariants (terms like $R^{2}, R^{\alpha \beta}, R_{\alpha \beta}, R^{\alpha \beta \gamma \delta}, R_{\alpha \beta \lambda \delta}$ etc.) or terms with scalar fields non minimally coupled to geometry (terms like $\phi^{2} R$ ). In general, one has to emphasize that terms like those are present in all the Approaches.

This approach is fine and agreeable but from the ontology of space time, as per me, a scalar field with a minimal energy cannot exist physically in this nature. Such space time cannot be stable and due to space time dynamics, mass will be created instead of remaining in energy state. Here basically mass and energy to be considered separate. All my earlier works emphasized on this fact. Mass will have a property of inertia where as energy do not contain that. At a glance it seems silly. But it makes lot of difference and describes the universe within diameter of Planck length. This present work stressed on that point and the space time dynamics applied to it in a classical way by which the dark energy has been 
calculated as un clustered. The dark matter can be calculated in the similar way but with the application of classical quantum mechanics. Thus the same phenomenology lies secretly within the Planck length as clustered as mentioned in the above approach.

But really it matters the curvature and demands for new terms with scalar field such as $\phi^{2} R$ curvature invariants like $R^{2}, R^{\alpha \beta}, R_{\alpha \beta}, R^{\alpha \beta \gamma \delta}, R_{\alpha \beta \lambda \delta}$ etc.

So some other information is lagging in defining curvature below Planck length to zero for which curvature becomes zero and velocity or momentum i.e. left side and right side of Einstein equations to be satisfied from Planck length to zero. The answer may lie in quantum mechanics and even beyond. My future works will explain it.

\subsection{Affect of This Phenomenon on Observations Involved in Experiments}

If this is the case, what is its affect on cosmological observation? The paper [10] explained its consequences on the observations. Specifically this work is based on the concept of "dark energy as a phenomenal effect of expansion" through which a theoretical value of Hubble's constant i.e. $H_{t h}$ has been introduced. It is a constant which is based on a postulate that the square root of ratio of Siva's constant " $K$ " and Hubble's constant " $H$ " is exactly equal to diameter of neutral Hydrogen atom. That is the exact theoretical value of Hubble's constant. This $H_{t h}$ is always constant. But space time conversions and change in velocity of light will affect the distance and velocity in Hubble's equation. Deviation factors in velocity, distance and the Hubble's constant have been calculated separately which are affected by change of velocity of light with expansion of space time. The observations are related to red shift. So the change factor in terms of Hubble's constant has been calculated with respect to red shift. Thus a factor which affects the theoretical Hubble's constant has been calculated to find the exact value of Hubble's constant that satisfies the experimental results.

The experimental results by plank, ACT (Atacama Cosmology Telescope), WMAP (Wilkinson Microwave Anisotropy Probe) etc. are at par with the theoretical result. Thus the ontology of space time introduced in this theoretical work supposed to be correct and leads to a new theory of origin of universe.

The extended paper [10] concluded that:

In Hubble's expanding universe formula $V=H d$, “ $V$ " is velocity of the receding galaxy and " $d$ " is the distance of that galaxy from observer. It says that " $V$ " is proportional to " $d$ " and " $H$ " is constant of proportionality. It is concluded that there is a theoretical value of " $H$ " called as $H_{t h}$ and it will vary with a factor $\frac{\Delta d}{\Delta V \times \Delta H}$

Thus the equation can be written as-

$$
V=\left(\frac{\Delta d}{\Delta V \times \Delta H}\right) H_{t h} \times d
$$


$\Delta d, \Delta H$ and $\Delta V$ are factors by which " $d$ ", " $H$ " and " $V$ " changes. These factors will vary with the distance from the observer. The above integrated factor changes with the red shift. We can find the exact value of Hubble's constant by red shift. It says that value in Mega parsecs depends on light velocity which changes with the distance. For present value of $1 \mathrm{Mpc}$, the Hubble's constant has been calculated as

$$
H=69.6 \mathrm{~km} \cdot \mathrm{s}^{-1} \cdot \mathrm{Mpc}^{-1} \text { or } 2.2555823860 \times 10^{-18} \mathrm{sec}^{-1}
$$

This is exact value of Hubble's constant.

Experimental values are supporting this theoretical frame work for calculating exact value of " $H$ ". So undoubtedly, the postulates, concepts and the new concept of space time in this theoretical frame work are correct. This may turn physics to a new dimension.

\subsection{Involvment of Geometric Derivation in “Dark Energy"}

Here is one more approach which is worth considering for review [11]. It is originated upon the basic that mass and energy are different aspects of space time depending upon space time dynamics. Most certainly quantum vacuum energy cannot be the usual gravitating mass-energy that astronomers measure. If it is not mass-energy, what other kind of energy could it be? Analysis of vacuum energy begins with the proposition that globally the big bang explanation of the observed expansion is wrong; a big bang is not the source of the expansion of our universe. There must be some other, as yet unknown, dynamic at work in our universe that causes it to expand. Knowing the cause of the expansion, we may be able to identify the rate of that dynamic as energy at both the macro and the micro level. Perhaps the expansion of our 3-D space is due to not just stretching, but to something that is being produced; the missing dynamic is the production of space. It is the author's contention that the source or building blocks for the production of our 3-D space can only be another higher-dimensional space. Instead of our 3-D space stretching in order to expand, a new vision of our beginning involves a new dynamic of spatial condensation that actually produces our expanding space. This new vision has our spatially 3-D closed universe as the surface of an expanding 4-D ball embedded in a pre-existing $\mathrm{m}-\mathrm{D}$ epi-space. A geometric derivation of the expansion rate of our universe, ignoring its mass energy contents, was shown equal to the expansion rate of a derivation in terms of the evolution of its contents.

But It is basically a disagreement to the ontology of the space time presented in this paper since in [11] author considered 3-D volume is different from 4-D volume. Basically present paper is based on the fact that volume of 3-D space and 4-D space both represent volume of space only and was firmly believed on space time conversions classically and space time dynamics is with the involvement of mass and energy.

\section{Conclusions}

1) It is proposed that the surface area of space time in spherical shape is vo- 
lume of its space and radius is its time. Space and time are interchangeable in an isolated system of space time in such a way that reduction in time will compensate for an increase of its surface area so that the volume of space will increase if time decreases in space time. Thus the total space time diameter will remain constant.

Space time equivalence has been given by equation

$$
t^{3}=3 d^{2}
$$

Where, " $t$ " is radius of "space time" and " $d$ " is diameter of "space time". Its surface area denotes three dimensional space.

2) Classical equations for space time have been derived. Equations are

$$
M=7.9905778 \times 10^{-17} \times d^{1 / 3}
$$

where " $M$ " is the mass associated with a space time diameter " $d$ " is

$$
\gamma d^{8 / 3}=1.526087946 \times 10^{-16}
$$

where $\gamma$ is the space time density associated with space time diameter " $d$ ".

3) Conversion of space, time and space time conversion into mass has been explained. This phenomenon interpreted the signal velocity (signal as defined by all transformations of relativity. As per relativity light is the signal for all transformations) in a different perspective and it is concluded that the signal velocity is proportional to the velocity of the receding galaxy interpreted by Hubble's expanding universe theory. It is formulated as Equation (21). This can be verified experimentally.

4) The same Equation (21) has been analysed further and emphasized that light velocity is changing with expansion of the universe. That equation concluded that the maximum velocity of the expansion of the universe is the maximum velocity of light. i.e. $8.9735835075 \times 10^{8} \mathrm{mt} \cdot \mathrm{sec}^{-1}$.

5) By postulating that the relation between Hubble's constant " $H$ " and Siva's constant " $K$ " is exactly equal to diameter of neutral Hydrogen atom, exact theoretical value of Hubble's constant i.e. $4.1394908592 \times 10^{-19} \mathrm{sec}^{-1}$. Necessity of that postulate has been discussed.

6) Changing velocity of light with expansion of the universe and its maximum velocity has been concluded by space time and mass conversions. The maximum velocity of light has been estimated as $8.9735835075 \times 10^{8} \mathrm{mt} \cdot \mathrm{sec}^{-1}$. Expected exact value or theoretical velocity of Hubble's constant has been calculated as $4.1394908592 \times 10^{-19} \mathrm{sec}^{-1}$. These two values have been interpreted with quantum particle photon and the energy associated with a single and first photon of the universe has been calculated. Thus the temperature of the universe when it is at the size of a photon (It is the first photon). This is nothing but Cosmic Microwave Background Radiation (CMBR) of the universe. The CMBR has been estimated as $2.68 \mathrm{~K}$ which is equal to the experimentally obtained result. These theoretical results at par with the experimental verification are an indication that the postulates used in this paper and the hypothesis proposed are on right path.

7) Dark energy is a consequence of the phenomena of the universe that elabo- 
rate mutual conversions of space, time, "space time" and matter. It concluded that observable mass energy density is $33.41 \%$ of the total mass energy density including hidden mass. The remaining $66.59 \%$ is in the form of space time which is necessary to produce this mass energy. Expansion is a phenomenon. Evolution of mass is a consequence of this phenomenon. Equation (34) shows the actual total mass energy density of the universe and Equation (35) is the mass energy density of the universe by considering density calculation based on expanding space alone. Thus dark energy is a consequence of missing physics in expanding universe theories. This can be observed experimentally as an effect that changes light velocity with expansion of the universe. This effect can be observed as interpreted by Equation (21). It shows that velocity of light is proportional to the velocity of the receding galaxy calculated by red shift observation.

8) Overall view of this concept is a solution for vacuum catastrophe of cosmology. It emphasizes the concept that space converts into time and "space time" converts into mass. It is against the notion of a concept that space expands with time. Thus its calculations showed that mass energy density of the universe is constant and observed as the matter is flying apart in space. It can be verified experimentally by observing light velocity with the expansion of the universe.

9) Same dark energy phenomena can explain dark matter also. The dark matter can be calculated as $27.21 \%$. Finally it can be concluded as dark energy contributes $66.59 \%$. In the present day observable universe, the mass energy of dark matter and conventional ordinary matter contributes $27.2 \%$ and $6.2 \%$ respectively.

\section{Conflicts of Interest}

The author declares no conflicts of interest regarding the publication of this paper.

\section{References}

[1] Padmanabhan, T. (2003) Cosmological Constant-The Weight of the Vacuum. Physics Reports, 380, 235-320. https://doi.org/10.1016/S0370-1573(03)00120-0

[2] Kodukula, S.P. (2017) Importance of Siva's Constant ' $K$ ' in Redefining Law of Gravitation as an Affect of Consciousness. International Journal of Theoretical and Mathematical Physics, 7, 155-158. http://article.sapub.org/10.5923.j.ijtmp.20170706.01.html

[3] Kodukula, S.P. (2017) New Perspective of Expanding Universe and Estimate for Mass of Universe by New Gravity Equation. International Journal of Astronomy, 6, 17-19. http://article.sapub.org/10.5923.j.astronomy.20170601.03.html

[4] Mohr, P.J., Newell, D.B. and Taylor, B.N. (2016) CODATA Recommended Values of the Fundamental Physical Constants: 2014. Journal of Physical and Chemical Reference Data, 45, 043102. https://doi.org/10.1063/1.4954402

[5] Bennett, C.L., Larson, D., Weiland, J.L. and Hinshaw, G. (2014) The 1\% Concordance Hubble Constant. The Astrophysical Journal, 794, 135.

https://doi.org/10.1088/0004-637X/794/2/135 https://iopscience.iop.org/article/10.1088/0004-637X/794/2/135/pdf

[6] Pr`sa, A., Harmanec, P., Torres, G., Mamajek, E., Asplund, M., Capitaine, N., 
Christensen-Dalsgaard, J., Depagne, 'E., Haberreiter, M., Hekker, S., Hilton, J., Kopp, G., Kostov, V., Kurtz, D.W., Laskar, J., Mason, B.D., Milone, E.F., Montgomery, M., Richards, M., Schmutz, W., Schou J. and Stewart, S.G. (2016) Nominalvalues for Selected Solar and Planetary Quantities: IAU 2015 Resolution B3.

https://doi.org/10.3847/0004-6256/152/2/41

http://www.iau.org/static/resolutions/IAU2015_English.pdf

[7] Kodukula, S.P. (2019) Values of Siva's Constant “K” for All Fundamental Forces-A Review on Spin, Threshold Time and Quantum Entanglement. Journal of Modern Physics, 10, 466-476. https://doi.org/10.4236/jmp.2019.104032

[8] Kodukula, S.P. (2017) Role of Observer \& Consciousness on Special Theory of Relativity and Its Influence on Kinetic Energy. International Journal of Physics, 5, 99-109. http://pubs.sciepub.com/ijp/5/4/1

[9] Corda, C. (2009) Interferometric Detection of Gravitational Waves: The Definitive Test for General Relativity. International Journal of Modern Physics D, 18, 2275-2282. https://doi.org/10.1142/S0218271809015904

[10] Kodukula, S.P. (2020) Theoretical Value of Hubble's Constant Is a Salient Feature of Experimental Results-New Insight in to Origin of Universe. Preprints, 2020, Article ID: 2020090452. https://www.preprints.org/manuscript/202009.0452/v1 https://doi.org/10.20944/preprints202009.0452.v1

[11] Charles, B. (2003) Leffert, A Resolution of the Vacuum Energy Problem. https://arxiv.org/ftp/astro-ph/papers/0308/0308014.pdf 